\title{
Long-Term Safety of Dapagliflozin in Older Patients with Type 2 Diabetes Mellitus: A Pooled Analysis of Phase IIb/III Studies
}

\author{
Paola Fioretto $^{1}$ - Traci A. Mansfield ${ }^{2}$ - Agata Ptaszynska ${ }^{3}$ - Yshai Yavin ${ }^{3,4}$. \\ Eva Johnsson ${ }^{5} \cdot$ Shamik Parikh $^{6}$
}

Published online: 29 June 2016

(c) The Author(s) 2016. This article is published with open access at Springerlink.com

\begin{abstract}
Objective To evaluate the 104-week safety of dapagliflozin in older patients with type 2 diabetes mellitus. Methods Pooled analysis assessing general safety (nine phase III studies $\leq 104$ weeks) and cardiovascular safety ( 21 phase IIb/III studies $\leq 208$ weeks) by age $(<65 ; \geq 65$; $\geq 75$ years). Patients with type 2 diabetes mellitus ( \pm background glucose-lowering therapy) received: dapagliflozin $10 \mathrm{mg}(n=2026)$ vs. placebo $(n=1956)$ (ninestudy pool); or dapagliflozin $(2.5-50 \mathrm{mg} ; n=5936) \mathrm{vs.}$ control (placebo/comparator) $(n=3403)$ (21-study pool). Results Adverse events (AEs) and discontinuations owing to AEs were more common in older vs. younger patients, and were more frequent with dapagliflozin than placebo (AEs: $<65$ years: 73.1 vs. $70.7 \%$; $\geq 65$ years: 77.4 vs. $73.1 \%$; $\geq 75$ years: 80.4 vs. $75.3 \%$, respectively; discontinuations: $<65$ years: 5.9 vs. $5.0 \%$; $\geq 65$ years: 14.4 vs.
\end{abstract}

The data in this manuscript were previously presented as an oral presentation at the 74th Scientific Sessions of the American Diabetes Association, San Francisco, June 13-17, 2014.

Electronic supplementary material The online version of this article (doi:10.1007/s40266-016-0382-1) contains supplementary material, which is available to authorized users.

Paola Fioretto

paola.fioretto@unipd.it

1 Department of Medicine, University of Padova, Via Giustiniani 2, Padua 35128, Italy

2 AstraZeneca, Fort Washington, PA, USA

3 Bristol-Myers Squibb, Princeton, NJ, USA

4 Present Address: Johnson \& Johnson, Raritan, NJ, USA

5 AstraZeneca, Gothenburg, Sweden

6 AstraZeneca, Gaithersburg, MD, USA
$12.2 \%$; $\geq 75$ years: 26.8 vs. $22.1 \%$, respectively); serious AE (SAE) frequency was similar $(<65$ years: 11.0 vs. $11.8 \%$; $\geq 65$ years: 20.0 vs. $20.2 \%$; $\geq 75$ years: 19.6 vs. $18.2 \%$, respectively). Hypoglycaemia frequency was similar across age groups and was higher with dapagliflozin than placebo ( $<65$ years: 18.0 vs. $13.4 \%$; $\geq 65$ years: 20.2 vs. $17.7 \%$; $\geq 75$ years: 17.5 vs. $16.9 \%$, respectively); major episodes were rare. Urinary tract infection frequency was similar between treatment groups in older patients, with no increase vs. younger patients $(<65$ years: 8.8 vs. $5.5 \%$; $\geq 65$ years: 8.1 vs. $7.6 \% ; \geq 75$ years: 8.2 vs. $9.1 \%$, respectively); urinary tract infection SAEs were rare. Genital infection AEs were more common with dapagliflozin, with no increase in older patients ( $<65$ years: 8.2 vs. $1.0 \%$; $\geq 65$ years: 6.6 vs. $0.9 \% ; \geq 75$ years: 7.2 vs. $0.0 \%$, respectively) and no SAEs. Volume reduction AEs were uncommon, with a higher frequency with dapagliflozin vs. placebo and in patients $\geq 75$ years $(<65$ years: 1.7 vs. $1.2 \%$; $\geq 65$ years: 2.3 vs. $1.7 \%$; $\geq 75$ years: 3.1 vs. $2.6 \%$, respectively). Dapagliflozin did not increase the risk of fractures ( $<65$ years: 1.1 vs. $1.1 \%$; $\geq 65$ years: 1.1 vs. $2.7 \%$; $\geq 75$ years: 1.0 vs. $2.6 \%$, respectively) or falls ( $<65$ years: 0.7 vs. $0.7 \%$; $\geq 65$ years: 0.6 vs. $2.1 \%$; $\geq 75$ years: 0.0 vs. $1.3 \%$, respectively), regardless of age. AEs of renal function were more common with dapagliflozin than placebo and increased with age $(<65$ years: 3.5 vs. $2.3 \%$; $\geq 65$ years: 14.0 vs. $7.9 \%$; $\geq 75$ years: 29.9 vs. $20.8 \%$, respectively). Most were non-serious small transient increases in serum creatinine. Dapagliflozin did not increase cardiovascular risk regardless of age [hazard ratio (95\% confidence interval) vs. control: $<65$ years: 0.726 $(0.473,1.114)$; $\geq 65$ years: $0.879 \quad(0.565,1.366)$; $\geq 75$ years: $0.950(0.345,2.617)$, respectively].

Conclusion Dapagliflozin treatment up to 104 weeks was well tolerated in older patients. Older dapagliflozin-treated 
patients had more renal AEs than placebo-treated patients; the majority of which were non-serious small transient changes in serum creatinine.

\section{Key Points}

The long-term safety of dapagliflozin was similar between older and younger patients, with comparable rates of hypoglycaemia, genital infections and urinary tract infections, low rates of volume reduction, and no increased risk of bone fractures or falls.

Adverse events of renal function were more frequent in patients receiving dapagliflozin and in older vs. younger patients; the majority of cases were nonserious and driven by small transient changes in serum creatinine.

Dapagliflozin did not increase the risk of cardiovascular disease in older or younger patients.

\section{Introduction}

The prevalence of type 2 diabetes mellitus (T2DM) is increasing globally, with the highest increases estimated amongst older populations [1]. A 4.5-fold increase in diabetes is predicted between 2005 and 2050 in people aged $\geq 65$ years, compared with a 3 -fold rise in the overall population [2].

Polypharmacy [3] and multiple comorbidities [4, 5] among older patients with T2DM present a number of challenges in terms of the clinical management of this patient group. In addition, pharmacokinetic and pharmacodynamic changes associated with ageing may increase the risk of adverse events (AEs) among this age group [5], including hypoglycaemia, volume reduction, impaired renal function and cardiovascular disease [6-8]. The increased susceptibility to hypoglycaemia among older people [9] is of particular concern because of the associated increased risk of falls and bone fractures [10].

Certain therapies may be less appropriate for some older patients, such as those associated with a higher risk of hypoglycaemia (e.g. sulphonylurea or insulin) [6]. Furthermore, complex dosing regimens for glycaemic control are not favoured. A once-daily oral medication regimen that can be taken at any time of day with or without food, with a low risk of hypoglycaemia and a well-characterised predictable safety profile, would be preferable.

Novel glucose-lowering therapies are required to address the unmet needs in the treatment of older patients with T2DM. Dapagliflozin is a highly selective sodium glucose co-transporter 2 (SGLT2) inhibitor that, in addition to reducing glycosylated haemoglobin $\left(\mathrm{HbA}_{1 \mathrm{c}}\right)$, body weight and systolic blood pressure [11-14], is associated with a low intrinsic risk of hypoglycaemia [16], and showed no increase in cardiovascular risk in a recent metaanalysis [15]. These benefits combined with its once-daily oral dosing regimen [17] suggest it may be a useful treatment in the management of older patients with T2DM. Identified risks include genital infections and, to a lesser extent, urinary tract infections and non-serious volumerelated events; all of which are consistent with the mechanism of action of dapagliflozin and are mostly of mild intensity [16].

Here, we present a pooled analysis of nine phase III studies to assess the long-term safety of dapagliflozin in older patients with T2DM. In addition, we present a pooled analysis of 21 phase IIb/III studies to assess the cardiovascular safety of dapagliflozin in older patients with T2DM.

\section{Methods}

\subsection{Patient Population}

Safety and efficacy were evaluated by age group $[<65, \geq 65$ and $\geq 75$ years (a subpopulation of the $\geq 65$ years age group; safety only)] in a placebo-controlled pool of nine double-blind phase III studies of $\leq 104$ weeks in duration, in which patients with T2DM received dapagliflozin $10 \mathrm{mg}$ $(n=2026)$ or placebo $(n=1956) \pm$ background glucoselowering therapy (Online Resource 1).

Cardiovascular events were evaluated by age group $[<65, \geq 65$ and $\geq 75$ years (a subpopulation of the $\geq 65$ years age group)], using data from a pre-specified cardiovascular meta-analysis of 21 phase IIb/III studies of $\leq 208$ weeks in duration, in which patients with T2DM received dapagliflozin $(2.5,5,10,20$ or $50 \mathrm{mg} ; n=5936)$ or control (placebo or comparator treatment; $n=3403) \pm$ background glucose-lowering therapy (Online Resource 2). Studies with at least one adjudicated cardiovascular event were included in the analysis.

These two different patient pools were each selected to address a specific objective. The nine-study pool was considered to be the most appropriate to assess general safety because all trials were long term (with at least 48 weeks of blinded study treatment) and placebo controlled. In contrast, the 21-study pool with high patient numbers was considered to be the most appropriate to assess the cardiovascular profile of dapagliflozin because cardiovascular events were relatively infrequent. The ninestudy safety pool examined dapagliflozin $10 \mathrm{mg}$ (the usual recommended clinical dose), whereas the 21-study 
cardiovascular pool examined all dapagliflozin doses $(2.5$, $5,10,20$ and $50 \mathrm{mg}$ ). Methods for each individual study in these pools have been published previously [11-14, 18-32]. All studies were conducted according to the principles of the Declaration of Helsinki and the requirements of Good Clinical Practice, and were approved by the relevant institutional review boards/ethics committees. All participants gave written informed consent.

\subsection{General Safety Outcomes}

\subsubsection{Adverse Events}

The frequency of overall AEs and serious AEs (SAEs) was evaluated according to age group up to 104 weeks.

\subsubsection{Adverse Events of Special Interest}

The proportion of patients with any event of hypoglycaemia was assessed by age and according to background medication, up to 104 weeks.

The frequency of AEs of genital infection, urinary tract infection (UTI), volume reduction (defined as hypotension, dehydration or hypovolaemia), bone fracture, fall and renal function were evaluated by age group up to 104 weeks, based on pre-defined lists of preferred terms from the Medical Dictionary of Regulatory Activities (version 15.1) (Online Resource 3). Reports of genital infections and UTIs were obtained through proactive questioning and spontaneous reporting. Calculation of estimated glomerular filtration rate (eGFR) used the Abbreviated Modification of Diet in Renal Disease Study equation [33]. Mean change from baseline in eGFR was determined for each age group up to 104 weeks, as were the maximum changes from baseline in serum creatinine in patients with at least one $\mathrm{AE}$ of renal function.

\subsubsection{Laboratory Values}

The frequency of marked abnormalities (MAs) in laboratory values was assessed for each age category up to 104 weeks. Laboratory assessments comprised the following: serum calcium, serum bicarbonate, serum potassium, serum magnesium, serum sodium, inorganic phosphorus, haematocrit, blood urea nitrogen and serum creatinine.

\subsection{Efficacy Outcomes}

\subsubsection{HbA $\mathrm{Hc}_{1 c}$ Body Weight and Systolic Blood Pressure}

Efficacy assessments for each age group comprised the mean change from baseline in $\mathrm{HbA}_{1 \mathrm{c}}$, the proportion of patients achieving an $\mathrm{HbA}_{1 \mathrm{c}}$ level of $<53 \mathrm{mmol} / \mathrm{mol}$
$(<7 \%)$ and the mean change from baseline in body weight and systolic blood pressure (SBP), up to 104 weeks.

\subsection{Analysis Method}

Analyses were conducted using descriptive statistics only. Comparisons with placebo were adjusted for differences in baseline values between groups. Analyses were performed using all available data regardless of rescue therapy, with the exception of hypoglycaemia and efficacy data [change from baseline in $\mathrm{HbA}_{1 \mathrm{c}}$, body weight and SBP, and the proportion of patients achieving an $\mathrm{HbA}_{1 \mathrm{c}}$ level of $<53 \mathrm{mmol} / \mathrm{mol}(<7 \%)$, which excluded data after the initiation of rescue therapy. Confidence intervals (CIs) for the proportion of patients achieving an $\mathrm{HbA}_{1 \mathrm{c}}$ level of $<53 \mathrm{mmol} / \mathrm{mol}(<7 \%)$ were estimated using logistic regression modelling for the difference between treatment groups.

\subsection{Cardiovascular Safety}

A meta-analysis was conducted according to age group to assess the cardiovascular risk for the pooled population of 21 phase IIb/III studies of $\leq 208$ weeks in duration, using the composite endpoint of 'cardiovascular death, myocardial infarction, stroke and hospitalisation for unstable angina'. All cardiovascular events were independently adjudicated in a blinded manner. The analyses were conducted without statistical hypothesis testing. Hazard ratios (HRs) and $95 \%$ CIs comparing total dapagliflozin doses $(2.5,5,10,20$ and $50 \mathrm{mg}$ ) with a control (placebo or comparator treatment) were calculated for each age group using the Cox proportional hazards model. An upper limit of the $95 \%$ CI of $<1$ (the null value) would indicate that dapagliflozin was associated with a lower cardiovascular risk than placebo, whereas a lower limit of the $95 \%$ CI of $>1$ would indicate that dapagliflozin was associated with a higher cardiovascular risk than placebo.

\section{Results}

\subsection{Patients}

Demographics and baseline characteristics for the nine pooled studies were generally balanced between the dapagliflozin and placebo groups in patients aged $<65$ and $\geq 65$ years (Table 1 ). Some differences were observed between the treatment groups in patients aged $\geq 75$ years (Table 1), which may reflect the small sample size for this age group ( $n=97$ for dapagliflozin vs. $n=77$ for placebo) and random variation within the $\geq 75$ years dataset rather than significant differences between the groups. 
Table 1 Demographic and baseline characteristics by age group

\begin{tabular}{|c|c|c|c|c|c|c|}
\hline & \multicolumn{2}{|l|}{ Age $<65$ years } & \multicolumn{2}{|l|}{ Age $\geq 65$ years } & \multicolumn{2}{|l|}{ Age $\geq 75$ years $^{\mathrm{a}}$} \\
\hline & $\begin{array}{l}\text { Dapagliflozin } \\
10 \mathrm{mg} \\
(n=1406)\end{array}$ & $\begin{array}{l}\text { Placebo } \\
(n=1301)\end{array}$ & $\begin{array}{l}\text { Dapagliflozin } \\
10 \mathrm{mg} \\
(n=620)\end{array}$ & $\begin{array}{l}\text { Placebo } \\
(n=655)\end{array}$ & $\begin{array}{l}\text { Dapagliflozin } \\
10 \mathrm{mg}(n=97)\end{array}$ & $\begin{array}{l}\text { Placebo } \\
(n=77)\end{array}$ \\
\hline Mean age, years (SD) & $54.6(7.5)$ & $54.8(7.5)$ & $69.9(4.0)$ & $69.7(4.0)$ & $77.1(1.8)$ & $77.8(2.8)$ \\
\hline Female, $n(\%)$ & $624(44.4)$ & $545(41.9)$ & $228(36.8)$ & $254(38.8)$ & $43(44.3)$ & $31(40.3)$ \\
\hline \multicolumn{7}{|l|}{ Race, $n(\%)$} \\
\hline Caucasian & $1179(83.9)$ & $1090(83.8)$ & $560(90.3)$ & $605(92.4)$ & $90(92.8)$ & $72(93.5)$ \\
\hline Asian & $96(6.8)$ & $94(7.2)$ & $35(5.6)$ & $26(4.0)$ & $6(6.2)$ & $3(3.9)$ \\
\hline Black/African American & $57(4.1)$ & $50(3.8)$ & $9(1.5)$ & $11(1.7)$ & $1(1.0)$ & $1(1.3)$ \\
\hline Other & $74(5.3)$ & $67(5.1)$ & $16(2.6)$ & $13(2.0)$ & 0 & $1(1.3)$ \\
\hline Mean time from T2DM diagnosis, years (SD) & $8.3(7.3)$ & $8.3(7.0)$ & $13.2(8.9)$ & $12.7(8.8)$ & $15.4(10.2)$ & $14.9(10.0)$ \\
\hline Mean $\mathrm{HbA}_{1 \mathrm{c}}, \mathrm{mmol} / \mathrm{mol}(\mathrm{SD})$ & $65(9.6)$ & $65(9.5)$ & $64(8.9)$ & $64(8.7)$ & $65(8.6)$ & $62(7.4)$ \\
\hline Mean FPG, mmol/L (SD) & $9.1(2.5)$ & $9.2(2.5)$ & $8.9(2.5)$ & $8.8(2.3)$ & $8.8(2.3)$ & $8.6(2.0)$ \\
\hline Mean weight, kg (SD) & $92.1(20.3)$ & $91.6(19.9)$ & $89.3(17.8)$ & $89.5(17.1)$ & $84.9(13.7)$ & $88.2(15.1)$ \\
\hline Mean BMI, kg/m² (SD) & $32.8(5.9)$ & $32.5(6.0)$ & $31.7(4.9)$ & $32.0(5.2)$ & $30.7(4.6)$ & $31.9(4.6)$ \\
\hline Mean systolic BP, mmHg (SD) & $130.5(15.2)$ & $130.1(15.0)$ & $135.7(14.7)$ & $135.5(13.9)$ & $138.7(14.5)$ & $133.3(13.8)$ \\
\hline \multicolumn{7}{|l|}{ Systolic BP, $n(\%)$} \\
\hline$\geq 130 \mathrm{mmHg}$ & $738(52.5)$ & $677(52.0)$ & $415(66.9)$ & $427(65.2)$ & $71(73.2)$ & $49(63.6)$ \\
\hline Mean eGFR, mL/min/1.73 $\mathrm{m}^{2}(\mathrm{SD})$ & $84.6(19.0)$ & $84.3(19.2)$ & $72.8(16.7)$ & $73.4(16.9)$ & $72.6(16.2)$ & $68.6(16.5)$ \\
\hline \multicolumn{7}{|l|}{ eGFR, $n(\%)$} \\
\hline$<30 \mathrm{~mL} / \mathrm{min} / 1.73 \mathrm{~m}^{2 \mathrm{~b}}$ & 0 & $1(0.1)$ & $1(0.2)$ & 0 & 0 & 0 \\
\hline$\geq 30$ to $<60 \mathrm{~mL} / \mathrm{min} / 1.73 \mathrm{~m}^{2 \mathrm{c}}$ & $117(8.3)$ & $108(8.3)$ & $134(21.6)$ & $141(21.5)$ & $22(22.7)$ & $22(28.6)$ \\
\hline$\geq 60$ to $<90 \mathrm{~mL} / \mathrm{min} / 1.73 \mathrm{~m}^{2 \mathrm{~d}}$ & $754(53.6)$ & $710(54.6)$ & $386(62.3)$ & $403(61.5)$ & $57(58.8)$ & $47(61.0)$ \\
\hline$\geq 90 \mathrm{~mL} / \mathrm{min} / 1.73 \mathrm{~m}^{2 \mathrm{e}}$ & $535(38.1)$ & $481(37.0)$ & $99(16.0)$ & $111(16.9)$ & $18(18.6)$ & $8(10.4)$ \\
\hline Mean serum creatinine, $\mu \mathrm{mol} / \mathrm{L}(\mathrm{SD})$ & $77.5(18.7)$ & $78.3(20.9)$ & $85.8(21.8)$ & $84.7(19.5)$ & $82.4(18.3)$ & $87.9(20.2)$ \\
\hline
\end{tabular}

Data available up to 104 weeks

$B M I$ body mass index, $B P$ blood pressure, $e G F R$ estimated glomerular filtration rate, $F P G$ fasting plasma glucose, $H b A_{l c}$ glycosylated haemoglobin, $S D$ standard deviation, $T 2 D M$ type 2 diabetes mellitus

${ }^{a}$ Subpopulation of the $\geq 65$ years age group

${ }^{\mathrm{b}}$ Severe renal impairment

${ }^{c}$ Moderate renal impairment

d Mild renal impairment

e Normal renal function

Older patients ( $\geq 65$ or $\geq 75$ years) had a relatively longer T2DM duration, higher systolic blood pressure and lower eGFR levels at baseline than younger patients ( $<65$ years) (Table 1).

\subsection{Overall Adverse Events}

Overall AEs and discontinuations owing to AEs were more common in patients aged $\geq 65$ and $\geq 75$ years than in patients aged $<65$ years, and were more frequent with dapagliflozin than placebo (Table 2). The frequency of SAEs was similar between the treatment groups across all ages, and more frequent in older vs. younger patients. In total, 30 deaths were reported in the nine pooled studies;
$18 / 2026(0.9 \%)$ in the dapagliflozin group and $12 / 1956$ $(0.6 \%)$ in the placebo group (Table 2$)$, none of which were considered related to treatment. Further information regarding these deaths is provided in Online Resource 4.

\subsection{Adverse Events of Special Interest}

\subsubsection{Hypoglycaemia}

The frequency of hypoglycaemia was similar between younger patients (aged $<65$ years) and older patients (aged $\geq 65$ or $\geq 75$ years), and more frequent with dapagliflozin than placebo, with major hypoglycaemic episodes being rare in all treatment groups (Table 2). In patients receiving 
Table 2 Adverse events by age group

\begin{tabular}{|c|c|c|c|c|c|c|}
\hline \multirow[t]{2}{*}{$n(\%)$} & \multicolumn{2}{|l|}{ Age $<65$ years } & \multicolumn{2}{|l|}{ Age $\geq 65$ years } & \multicolumn{2}{|l|}{ Age $\geq 75$ years $^{\mathrm{a}}$} \\
\hline & $\begin{array}{l}\text { Dapagliflozin } \\
10 \mathrm{mg}(n=1406)\end{array}$ & $\begin{array}{l}\text { Placebo } \\
(n=1301)\end{array}$ & $\begin{array}{l}\text { Dapagliflozin } \\
10 \mathrm{mg}(n=620)\end{array}$ & $\begin{array}{l}\text { Placebo } \\
(n=655)\end{array}$ & $\begin{array}{l}\text { Dapagliflozin } \\
10 \mathrm{mg}(n=97)\end{array}$ & $\begin{array}{l}\text { Placebo } \\
(n=77)\end{array}$ \\
\hline \multicolumn{7}{|l|}{ AEs } \\
\hline All & $1028(73.1)$ & $920(70.7)$ & $480(77.4)$ & $479(73.1)$ & $78(80.4)$ & $58(75.3)$ \\
\hline $\mathrm{AE}$ leading to discontinuation & $83(5.9)$ & $65(5.0)$ & $89(14.4)$ & $80(12.2)$ & $26(26.8)$ & $17(22.1)$ \\
\hline Any $\mathrm{SAE}^{\mathrm{b}}$ & $154(11.0)$ & 154 (11.8) & $124(20.0)$ & $132(20.2)$ & 19 (19.6) & $14(18.2)$ \\
\hline Deaths & $7(0.5)$ & $7(0.5)$ & $11(1.8)$ & $5(0.8)$ & $2(2.0)$ & $1(1.3)$ \\
\hline \multicolumn{7}{|l|}{ AEs of special interest } \\
\hline \multicolumn{7}{|l|}{ Hypoglycaemia } \\
\hline All & $253(18.0)$ & $174(13.4)$ & $125(20.2)$ & $116(17.7)$ & $17(17.5)$ & $13(16.9)$ \\
\hline Major episodes $^{\mathrm{c}}$ & $2(0.1)$ & $2(0.2)$ & $2(0.3)$ & 0 & 0 & 0 \\
\hline \multicolumn{7}{|l|}{ Genital infection } \\
\hline All & $115(8.2)$ & $13(1.0)$ & $41(6.6)$ & $6(0.9)$ & $7(7.2)$ & 0 \\
\hline Female & $78(12.5)$ & $12(2.2)$ & $20(8.8)$ & $3(1.2)$ & $6(14.0)$ & 0 \\
\hline Male & $37(4.7)$ & $1(0.1)$ & $21(5.4)$ & $3(0.7)$ & $1(1.9)$ & 0 \\
\hline SAEs $^{\mathrm{b}}$ & 0 & 0 & 0 & 0 & 0 & 0 \\
\hline \multicolumn{7}{|l|}{ Urinary tract infection } \\
\hline All & $124(8.8)$ & $71(5.5)$ & $50(8.1)$ & $50(7.6)$ & $8(8.2)$ & $7(9.1)$ \\
\hline Female & $93(14.9)$ & $52(9.5)$ & $28(12.3)$ & $34(13.4)$ & $4(9.3)$ & $5(16.1)$ \\
\hline Male & $31(4.0)$ & $19(2.5)$ & $22(5.6)$ & $16(4.0)$ & $4(7.4)$ & $2(4.3)$ \\
\hline SAEs $^{\mathrm{b}}$ & $3(0.2)$ & $2(0.2)$ & $2(0.3)$ & $1(0.2)$ & $1(1.0)$ & 0 \\
\hline Pyelonephritis & $2(0.1)$ & $1(0.1)$ & $1(0.2)$ & $2(0.3)$ & 0 & 0 \\
\hline \multicolumn{7}{|l|}{ Volume reduction $^{\mathrm{d}}$} \\
\hline All & $24(1.7)$ & $16(1.2)$ & $14(2.3)$ & $11(1.7)$ & $3(3.1)$ & $2(2.6)$ \\
\hline SAEs $^{\mathrm{b}}$ & $1(<0.1)$ & $3(0.2)$ & $2(0.3)$ & $3(0.4)$ & 0 & $1(1.3)$ \\
\hline Syncope & $1(<0.1)$ & $2(0.2)$ & $1(0.2)$ & $3(0.5)$ & 0 & $1(1.3)$ \\
\hline Circulatory collapse & 0 & $1(<0.1)$ & $1(0.2)$ & 0 & 0 & 0 \\
\hline \multicolumn{7}{|l|}{ Renal function } \\
\hline All & $49(3.5)$ & $30(2.3)$ & $87(14.0)$ & $52(7.9)$ & $29(29.9)$ & $16(20.8)$ \\
\hline SAEs $^{\mathrm{b}}$ & $4(0.3)$ & $1(0.1)$ & $1(0.2)$ & $2(0.3)$ & $1(1.3)$ & 0 \\
\hline
\end{tabular}

Data available up to 104 weeks. All results included data after rescue therapy, apart from hypoglycaemia for which data after rescue therapy were excluded

$A E$ adverse event, $N$ total number of patients, $n$ number of patients with an AE, $S A E$ serious adverse event

a Subpopulation of the $\geq 65$ years age group

b Based on the International Conference on Harmonisation of Technical Requirements for Registration of Pharmaceuticals for Human Use definition

c Symptomatic episode requiring external assistance, with a capillary or plasma glucose value $<54 \mathrm{mg} / \mathrm{dL}$ ( $3 \mathrm{mmol} / \mathrm{L})$ and prompt recovery after glucose or glucagon administration

d Hypotension, dehydration or hypovolaemia

dapagliflozin as monotherapy or in combination with metformin, a thiazolidinedione or a dipeptidyl peptidase- 4 inhibitor, the frequency of hypoglycaemia with dapagliflozin was similar to placebo for all age groups (Online Resource 5). Hypoglycaemia was more common in studies where treatment was combined with insulin or a sulphonylurea, with comparatively higher rates in dapagliflozintreated patients than in placebo-treated patients.

\subsubsection{Genital Infection and Urinary Tract Infection}

Events of genital infection occurred more frequently with dapagliflozin than placebo but did not substantially differ between age groups (Table 2). Events were more common among female patients regardless of age. All AEs of genital infection were reported to be mild or moderate in intensity and responded to standard treatment. 
Although AEs of UTI were more common with dapagliflozin than placebo treatment in patients aged $<65$ years, frequencies were similar between treatment groups in patients aged $\geq 65$ and $\geq 75$ years (Table 2) and did not increase with age. AEs of UTI were more common among female patients. Most events of UTI were reported to be of mild or moderate intensity, and SAEs were rare for all age groups. Few cases of pyelonephritis were reported with dapagliflozin or placebo, regardless of age (Table 2).

\subsubsection{Volume Reduction}

The frequency of AEs of volume reduction was low in both treatment arms across all age groups, with a small increase in events with dapagliflozin and in patients aged $\geq 75$ years (Table 2). Most volume reduction AEs were reported as hypotension or syncope. The majority of AEs of volume reduction were mild or moderate in intensity, did not require intervention and had resolved within 28 days [number of events (\%) with a duration $>28$ days: $<65$ years: $n=8 / 30(26.7 \%)$ vs. $n=0 / 17(0.0 \%)$; $\geq 65$ years: $n=2 / 17 \quad(11.8 \%)$ vs. $n=0 / 12 \quad(0.0 . \%)$; $\geq 75$ years: $n=1 / 5(20.0 \%)$ vs. $n=0 / 2(0.0 \%)$, with dapagliflozin vs. placebo, respectively]. Few SAEs of volume reduction were reported in either treatment group, without any obvious imbalance between groups; most SAEs were reported as syncope (Table 2).

\subsubsection{Renal Function}

Renal function remained stable over time in patients treated with dapagliflozin (Fig. 1). In younger patients, eGFR returned to baseline within a few weeks of treatment after a small early reduction with dapagliflozin vs. placebo $(-4.0$ vs. $0.1 \mathrm{~mL} / \mathrm{min} / 1.73 \mathrm{~m}^{2}$ at week 1 , respectively). In older patients, the initial reduction in eGFR was of a similar magnitude to younger patients ( $\geq 65$ years: -4.7 vs. $0.9 \mathrm{~mL} / \mathrm{min} / 1.73 \mathrm{~m}^{2}$ at week 1 ; $\geq 75$ years: -5.2 vs. $0.4 \mathrm{~mL} / \mathrm{min} / 1.73 \mathrm{~m}^{2}$ at week 4 , with dapagliflozin and placebo, respectively); however, recovery of eGFR appeared slower, and levels remained below baseline but were stable up to 102 weeks. Interestingly, after the first 24 weeks of treatment, eGFR values were superimposable in patients receiving dapagliflozin and placebo (Fig. 1).

AEs of renal function were more common with dapagliflozin (Table 2) and the frequency of events increased with age in both treatment groups. Most AEs of renal function were reports of increased creatinine levels, reduced creatinine clearance, renal impairment or reduced eGFR. Few SAEs of renal function were reported.
The distribution of serum creatinine changes in patients with a reported $\mathrm{AE}$ of renal function was similar between the treatment groups in patients aged $<65$ and $\geq 65$ years, with a higher frequency of minor, transient serum creatinine changes ( 9 to $\leq 27 \mu \mathrm{mol} / \mathrm{L}$ ) reported with dapagliflozin in patients aged $\geq 75$ years (Fig. 2).

MAs of serum creatinine of $\geq 133 \mu \mathrm{mol} / \mathrm{L}$ were more frequent in older vs. younger patients, with a similar frequency between the dapagliflozin and placebo groups (Online Resource 6). MAs of serum creatinine of $\geq 221 \mu \mathrm{mol} / \mathrm{L}$ were uncommon in all age groups. MAs of blood urea nitrogen were balanced between the treatment groups regardless of age.

\subsection{Bone Fractures, Falls and Non-renal Laboratory Abnormalities}

The frequency of fractures was numerically similar or lower with dapagliflozin vs. placebo for all age groups $[<65$ years: $16(1.1 \%)$ vs. $14(1.1 \%)$; $\geq 65$ years: $7(1.1 \%)$ vs. 18 $(2.7 \%)$; $\geq 75$ years: $1(1.0 \%)$ vs. 2 (2.6\%), respectively]. Dapagliflozin was not associated with an increased risk of falls compared with placebo in any age category $[<65$ years: $10(0.7 \%)$ vs. $9(0.7 \%)$; $\geq 65$ years: $4(0.6 \%)$ vs. 14 $(2.1 \%) ; \geq 75$ years: 0 vs. 1 (1.3\%), respectively).

Electrolyte abnormalities were infrequent, and MAs in serum calcium, serum bicarbonate, serum magnesium and serum sodium were balanced between the treatment arms and across age groups (Online Resource 6). Dapagliflozin was associated with a higher frequency of serum potassium MAs of $\geq 6.0 \mathrm{mmol} / \mathrm{L}$ than placebo in older but not younger patients (Online Resource 6). No cases of serum potassium MAs of $\leq 2.5 \mathrm{mmol} / \mathrm{L}$ were reported. MAs of inorganic phosphorus $(\geq 1.81 \mathrm{mmol} / \mathrm{L})$ were more common with dapagliflozin and were more common in older vs. younger patients (Online Resource 6). MAs of haematocrit $>55 \%$ were more common with dapagliflozin regardless of age.

\subsection{Efficacy Outcomes}

\subsection{1 $\mathrm{HbA}_{1 \mathrm{c}}$}

Dapagliflozin was associated with a greater reduction from baseline in $\mathrm{HbA}_{1 \mathrm{c}}$ up to 104 weeks compared with placebo in both patients aged $<65$ and those aged $\geq 65$ years (Online Resource 7). A significantly greater proportion of patients achieved an $\mathrm{HbA}_{1 \mathrm{c}}$ value of $<53 \mathrm{mmol} / \mathrm{mol}$ $(<7 \%)$, with dapagliflozin vs. placebo in both age groups ( $<65$ years: 25.6 vs. $15.2 \%$; $\geq 65$ years: 18.2 vs. $12.3 \%$ ). As such, the efficacy of dapagliflozin in reducing $\mathrm{HbA}_{1 \mathrm{c}}$ was similar in older vs. younger patients. 
Fig. 1 Mean changes from baseline in eGFR over time by age group. Data available up to 102 weeks; includes data after rescue therapy. $B L$ baseline, $C I$ confidence interval, $e G F R$ estimated glomerular filtration rate, $S D$ standard deviation.

${ }^{\text {a }}$ Subpopulation of the $\geq 65$ years age group
eGFR $<65$ years

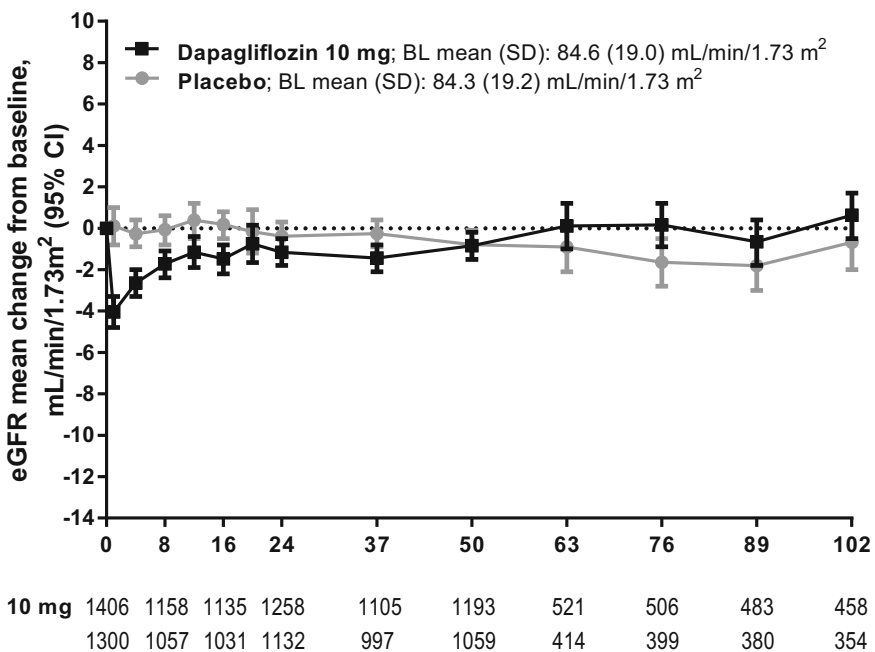

$\begin{array}{lllllllllll}\text { Dapagliflozin } 10 \text { mg } & 1406 & 1158 & 1135 & 1258 & 1105 & 1193 & 521 & 506 & 483 & 458 \\ & 1300 & 1057 & 1031 & 1132 & 997 & 1059 & 414 & 399 & 380 & 354\end{array}$

eGFR $\geq 65$ years

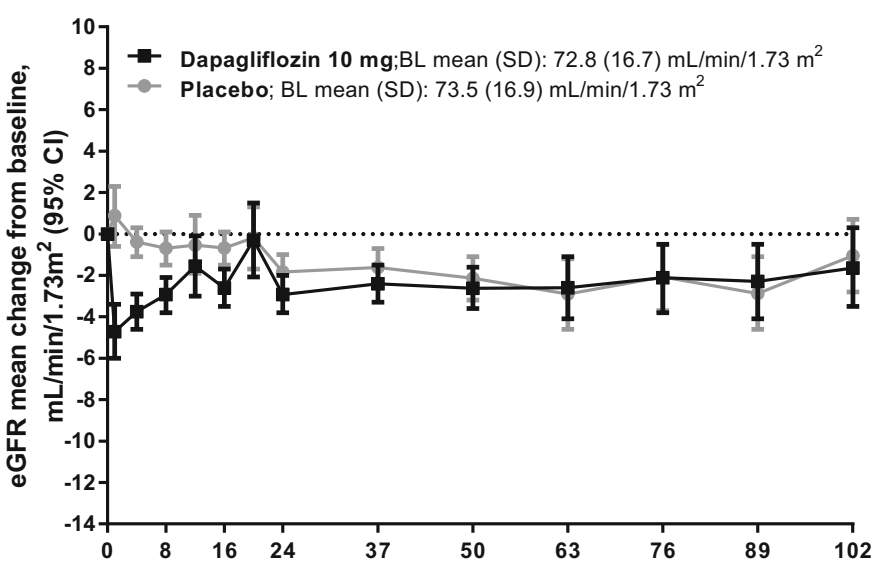

$\mathrm{N}^{\mathrm{o}}$ patients

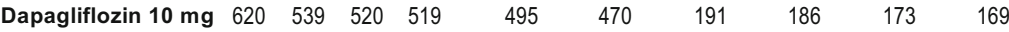

Placebo

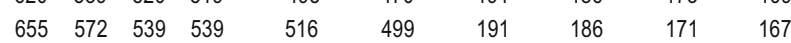

eGFR $\geq 75$ years $^{a}$

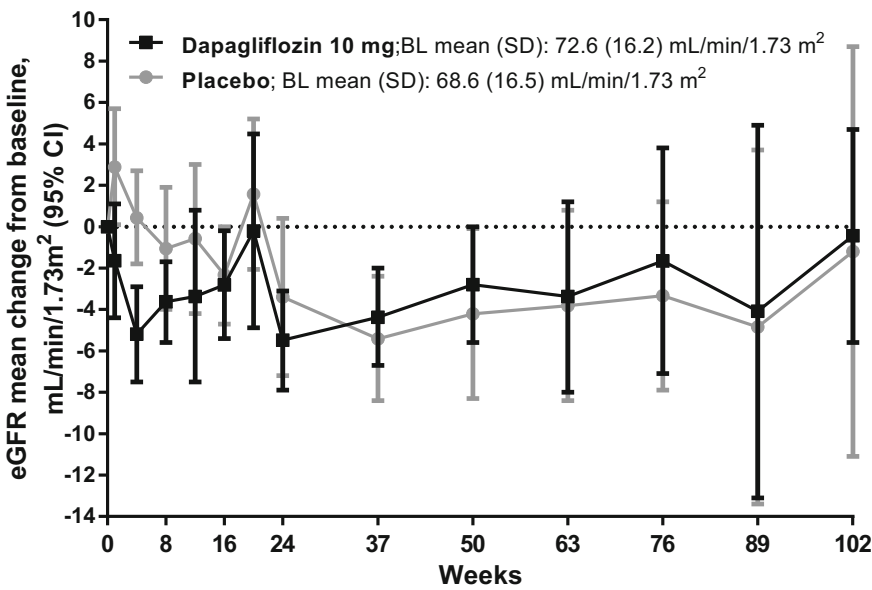

$N^{\circ}$ patients

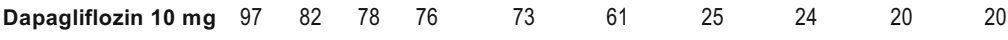

$\begin{array}{lllllllllll}\text { Placebo } & 77 & 71 & 62 & 58 & 59 & 53 & 16 & 15 & 13 & 10\end{array}$ 

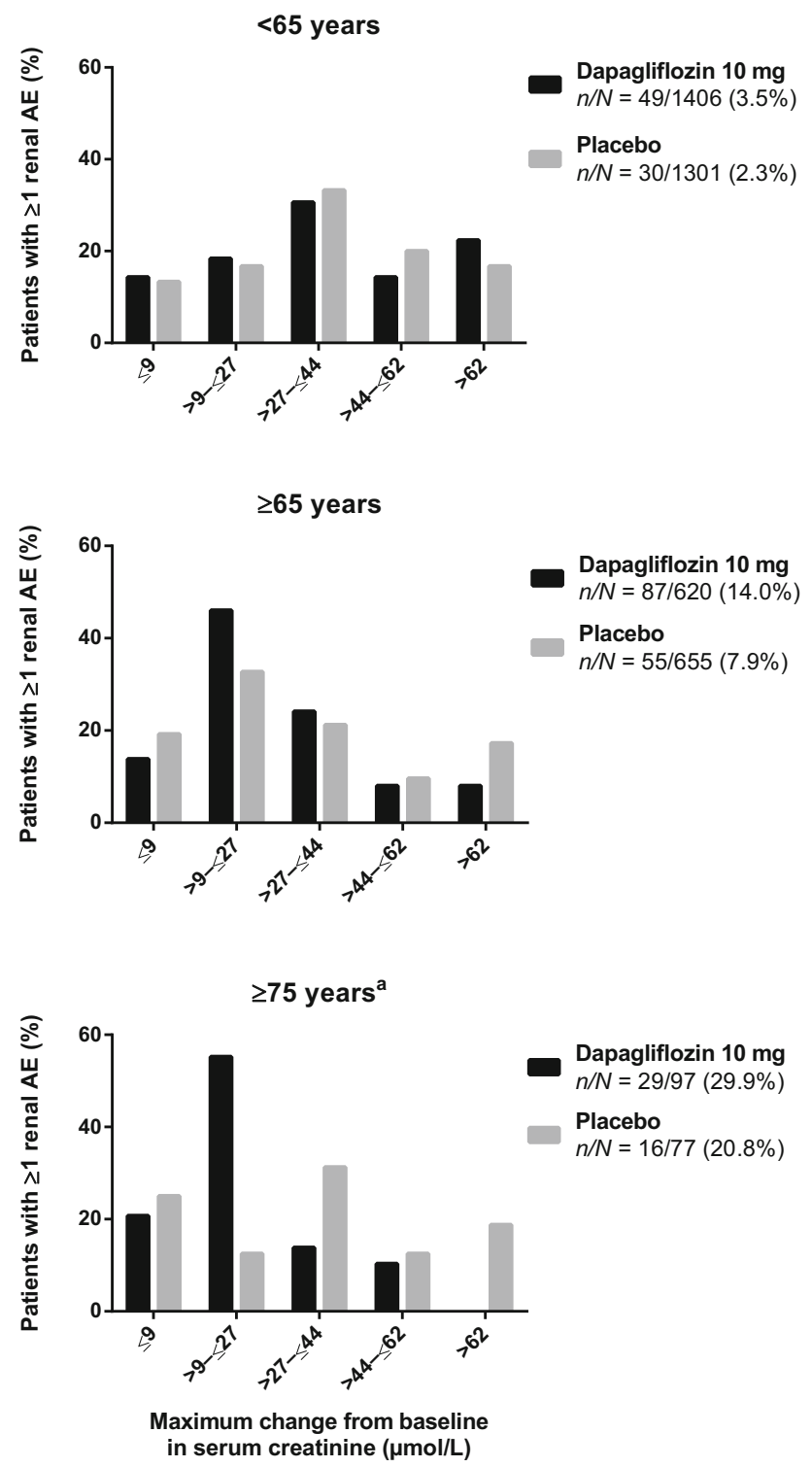

Fig. 2 Maximum change from baseline in serum creatinine in patients with AEs of renal function, by age group. Data show the proportion of patients with a maximum change from baseline in serum creatinine in the range indicated. Data available up to 102 weeks; $n$ is the number of patients with an AE of renal function; $N$ is the number of treated patients. Includes data after rescue therapy. $A E$ adverse event. ${ }^{\text {a }}$ Subpopulation of the $\geq 65$ years age group

\subsubsection{Body Weight and Systolic Blood Pressure}

For both patients aged $<65$ years and those aged $\geq 65$ years, reductions in body weight were greater with dapagliflozin than with placebo up to 104 weeks (Online Resource 8). SBP reduction was more pronounced with dapagliflozin compared with placebo in both age categories, although absolute changes were generally small (Online Resource 9).

\subsection{Cardiovascular Risk}

In the 21-study cardiovascular meta-analysis of $\leq 208$ weeks [dapagliflozin, $n=5936$ vs. control (placebo or comparator treatment), $n=3403]$, the HR for the composite endpoint of 'cardiovascular death, myocardial infarction, stroke and hospitalisation for unstable angina' was $<1$ for dapagliflozin vs. control in all age groups (Fig. 3), indicating that dapagliflozin does not increase the risk of cardiovascular disease in these patients.

\section{Discussion}

This pooled analysis of nine phase III studies investigated the safety profile of dapagliflozin across age subgroups. Dapagliflozin $10 \mathrm{mg}$ was generally well tolerated in both younger and older patients with T2DM during the 2-year treatment period. As expected, dapagliflozin was shown to reduce $\mathrm{HbA}_{1 \mathrm{c}}$, body weight and SBP compared with placebo across age groups.

Despite the fact that older patients are at higher risk of hypoglycaemia [8], rates were generally comparable across all ages, with a higher frequency in the dapagliflozin group compared with the placebo group. Of note, older patients treated with dapagliflozin either as monotherapy or in combination with metformin, a thiazolidinedione or a dipeptidyl peptidase-4 inhibitor had a similar risk of hypoglycaemia as placebo, in line with previous studies showing that dapagliflozin is associated with a low intrinsic hypoglycaemic risk [32, 34].

The increased risk of genital infections with dapagliflozin $10 \mathrm{mg}$ relative to placebo is consistent with previous observations of SGLT2 inhibitors [35], but frequencies did not increase in older patients. These events are typical for diabetes and the risk, presentation and treatment options are familiar to both physicians and patients [36]. Furthermore, despite the fact that older people are more susceptible to UTIs [37], rates did not increase in older patients in the current analysis.

Dapagliflozin has a mild diuretic effect, and therefore volume-related events are of special interest, especially in older patients [17]. Older patients have an increased propensity to develop volume reduction, which may be attributed to changes in body composition with ageing, leading to impairments in volume regulation and dysregulation of fluid intake [38]. Volume reduction may also be exacerbated by the diuretic treatments taken by many older patients [39]. AEs of volume reduction were more frequent with dapagliflozin $10 \mathrm{mg}$ than placebo, and were higher in patients aged $\geq 75$ years in both treatment groups. Most volume reduction events with dapagliflozin were reported 


\begin{tabular}{|c|c|c|c|c|}
\hline Age group & $\begin{array}{l}\text { DAPA, } \\
n / N\end{array}$ & $\begin{array}{c}\text { Control, } \\
n / N\end{array}$ & DAPA $\stackrel{\text { Favours }}{\longleftarrow} \stackrel{\text { Control }}{\longrightarrow}$ & HR vs. control $(95 \% \mathrm{Cl})$ \\
\hline$<65$ years & $48 / 4309$ & $42 / 2292$ & $\longmapsto$ & $0.726(0.473,1.114)$ \\
\hline$\geq 65$ years & $47 / 1390$ & $39 / 948$ & $\longmapsto$ & $0.879(0.565,1.366)$ \\
\hline \multirow[t]{3}{*}{$\geq 75$ years $^{b}$} & 9/204 & $7 / 118$ & $\longrightarrow$ & $0.950(0.345,2.617)$ \\
\hline & \multirow{2}{*}{\multicolumn{2}{|c|}{0.1}} & 1 & 10 \\
\hline & & & IRR (95\% CI) & \\
\hline
\end{tabular}

Fig. 3 Cardiovascular composite endpoint ${ }^{\mathrm{a}}$ by age. Data available up to 208 weeks. An HR upper $95 \%$ CI of $<1$ would indicate that dapagliflozin was associated with longer cardiovascular compositefree survival than control. $n$ is the number of patients who experienced a cardiovascular composite endpoint; $N$ is the total

as hypotension with no intervention required, which is consistent with previous reports [16], and the vast majority of events were mild or moderate in intensity.

Renal function in older and younger patients treated with dapagliflozin remained stable over time, with small transient reductions in eGFR relative to placebo early on in treatment. Similar to observations made in patients with impaired renal function [25], eGFR levels did not completely return to baseline levels in older patients, after an initial small drop in eGFR, which is consistent with the mechanism of action of dapagliflozin. These early small eGFR decreases followed by long-term stability across age groups are likely related to tubuloglomerular feedback responses and/or blood pressure reduction [40], and should not lead to discontinuation of dapagliflozin treatment. Ageing is associated with a progressive decline in eGFR [38] and slower renal function recovery times [41], which may be attributed to an impaired capacity for autoregulation [42]. In the current analysis, the fact that eGFR levels did not fully recover to baseline values in older patients, irrespective of treatment group, is in keeping with the reduced renal function in this population.

In view of the diuretic effect of dapagliflozin [26] and some transient volume shifts, it is not surprising to see elevations in serum creatinine, especially in patients with renal impairment. AEs of renal function were more commonly reported in patients treated with dapagliflozin $10 \mathrm{mg}$ than placebo and were more frequent in older patients than in younger patients, consistent with an increased susceptibility to AEs of renal function among this age group. Owing to their small magnitude, these changes were not considered to be clinically meaningful. Dapagliflozin was not associated with any electrolyte changes typical of number of treated patients. $C I$ confidence interval, DAPA dapagliflozin, $H R$ hazard ratio. ${ }^{\mathrm{a} C a r d i o v a s c u l a r}$ death, myocardial infarction, stroke and hospitalisation for unstable angina. ' $\mathrm{S} u b p o p u l a t i o n$ of the $\geq 65$ years age group

conventional diuretics [43], and did not increase the frequency of fractures or falls, regardless of age. Previous studies have shown that dapagliflozin does not affect bone mineral density or markers of bone formation and resorption [44].

Dapagliflozin did not increase the risk of hyperkalaemia compared with placebo in younger patients (aged $<65$ years), although a small increase was reported in older patients (aged $\geq 65$ and $\geq 75$ years). However, the majority of cases of hyperkalaemia were not reported as AEs. A previous pooled analysis in patients with T2DM demonstrated that dapagliflozin did not increase the risk of hyperkalaemia [16], although the majority of patients in this analysis $(\sim 70 \%)$ were aged $<65$ years. The higher frequency of hyperkalaemia in older patients observed in the current analysis may be partly related to an age-related decline in renal function and the increased use of potassium-altering medications among many older people compared with younger people [45].

Older patients with T2DM are at considerable risk of cardiovascular complications [5]. The data presented here provide reassurance regarding the cardiovascular safety of dapagliflozin and suggest a potential cardiovascular benefit, which is consistent with the multifactorial beneficial effects on cardiovascular risk factors associated with SGLT2 inhibitors. Indeed, a recent trial in patients with T2DM and established cardiovascular disease demonstrated significant improvements in cardiovascular outcomes with the SGLT2 inhibitor empagliflozin [46]. Previous studies indicate that dapagliflozin has positive effects on a number of cardiovascular risk factors including weight loss and blood pressure reduction [15, 47-49], which will be further investigated in a large randomised 
placebo-controlled trial assessing cardiovascular outcomes in a broad population of more than 17,000 patients with T2DM and established cardiovascular disease or in patients having multiple cardiovascular risk factors (DECLARE; clinicaltrials.gov ID NCT01730534).

Limitations to this pooled analysis include the low patient numbers in the $\geq 75$ years age group $(n=174)$ in comparison with the other two age categories ( $\geq 65$ years, $n=1275 ;<65$ years, $n=2707$ ). In addition, although the number of patients included in the cardiovascular metaanalysis was larger $(n=9339)$, the total number of cardiovascular events reported was low $(n=192)$. The generalisability of the results of this study is limited, as data on the functional status of the patients were not available, and the vast majority of patients in the analysis $(>80 \%)$ were Caucasian.

\section{Conclusion}

This pooled post hoc analysis suggests a favourable safety profile for dapagliflozin in older patients (aged $\geq 65$ and $\geq 75$ years) with T2DM. Safety over 2 years of treatment with dapagliflozin $10 \mathrm{mg}$ was similar between older and younger patients, with comparable rates of hypoglycaemia, genital infections and urinary tract infections, low rates of volume reduction and no increased risk of bone fractures. AEs of renal function were more frequent in older patients, the majority of which were non-serious and driven by small transient changes in serum creatinine.

Acknowledgments Medical writing support was provided by Helen Brereton of inScience Communications, Springer Healthcare Ltd, London, UK, funded by AstraZeneca. John Xu (AstraZeneca, Gaithersburg, MD, USA) provided statistical support.

\section{Compliance with Ethical Standards}

Conflict of interest PF served as a speaker and a consultant for AstraZeneca, Janssen and Boehringer Ingelheim. TM is an employee and shareholder of AstraZeneca, and a shareholder of Bristol-Myers Squibb. AP is an employee and shareholder of Bristol-Myers Squibb. YY was a previous employee of Bristol-Myers Squibb and is a shareholder of Bristol-Myers Squibb. EJ is an employee and shareholder of AstraZeneca. SM is an employee and shareholder of AstraZeneca.

Ethical Approval All studies included in this pooled analysis were performed in accordance with the ethical standards of the institutional and/or national research committee and with the 1964 Helsinki Declaration and its later amendments or comparable ethical standards.

Informed Consent Informed consent was obtained from all individual participants included in the studies.

Funding Funding for this post hoc analysis and for the medical writing support was provided by AstraZeneca.
Open Access This article is distributed under the terms of the Creative Commons Attribution-NonCommercial 4.0 International License (http://creativecommons.org/licenses/by-nc/4.0/), which permits any noncommercial use, distribution, and reproduction in any medium, provided you give appropriate credit to the original author(s) and the source, provide a link to the Creative Commons license, and indicate if changes were made.

\section{References}

1. Boyle JP, Honeycutt AA, Narayan KM, Hoerger TJ, Geiss LS, Chen H, et al. Projection of diabetes burden through 2050: impact of changing demography and disease prevalence in the U.S. Diabetes Care. 2001;24(11):1936-40.

2. Narayan KM, Boyle JP, Geiss LS, Saaddine JB, Thompson TJ. Impact of recent increase in incidence on future diabetes burden: U.S., 2005-2050. Diabetes Care. 2006;29(9):2114-6.

3. Johnell K, Klarin I. The relationship between number of drugs and potential drug-drug interactions in the elderly: a study of over 600,000 elderly patients from the Swedish Prescribed Drug Register. Drug Saf. 2007;30(10):911-8.

4. Valencia WM, Florez H. Pharmacological treatment of diabetes in older people. Diabetes Obes Metab. 2014;16(12):1192-203.

5. Kirkman MS, Briscoe VJ, Clark N, Florez H, Haas LB, Halter JB, et al. Diabetes in older adults: a consensus report. J Am Geriatr Soc. 2012;60(12):2342-56.

6. Inzucchi SE, Bergenstal RM, Buse JB, Diamant M, Ferrannini E, Nauck M, et al. Management of hyperglycemia in type 2 diabetes: a patient-centered approach: position statement of the American Diabetes Association (ADA) and the European Association for the Study of Diabetes (EASD). Diabetes Care. 2012;35(6):1364-79.

7. Rastegar A, Soleimani M. Hypokalaemia and hyperkalaemia. Postgrad Med J. 2001;77(914):759-64.

8. Bramlage P, Gitt AK, Binz C, Krekler M, Deeg E, Tschope D. Oral antidiabetic treatment in type-2 diabetes in the elderly: balancing the need for glucose control and the risk of hypoglycemia. Cardiovasc Diabetol. 2012;11:122.

9. Miller ME, Bonds DE, Gerstein HC, Seaquist ER, Bergenstal RM, Calles-Escandon J, et al. The effects of baseline characteristics, glycaemia treatment approach, and glycated haemoglobin concentration on the risk of severe hypoglycaemia: post hoc epidemiological analysis of the ACCORD study. BMJ. 2010;340:b5444.

10. Malabu UH, Vangaveti VN, Kennedy RL. Disease burden evaluation of fall-related events in the elderly due to hypoglycemia and other diabetic complications: a clinical review. Clin Epidemiol. 2014;6:287-94.

11. Nauck MA, Del Prato S, Meier JJ, Duran-Garcia S, Rohwedder $\mathrm{K}$, Elze M, et al. Dapagliflozin versus glipizide as add-on therapy in patients with type 2 diabetes who have inadequate glycemic control with metformin: a randomized, 52-week, double-blind, active-controlled noninferiority trial. Diabetes Care. 2011;34(9): 2015-22.

12. Rosenstock J, Vico M, Wei L, Salsali A, List JF. Effects of dapagliflozin, an SGLT2 inhibitor, on $\mathrm{HbA}(1 \mathrm{c})$, body weight, and hypoglycemia risk in patients with type 2 diabetes inadequately controlled on pioglitazone monotherapy. Diabetes Care. 2012;35(7):1473-8.

13. Strojek K, Yoon KH, Hruba V, Elze M, Langkilde AM, Parikh S. Effect of dapagliflozin in patients with type 2 diabetes who have inadequate glycaemic control with glimepiride: a randomized, 
24-week, double-blind, placebo-controlled trial. Diabetes Obes Metab. 2011;13(10):928-38.

14. Wilding JP, Woo V, Soler NG, Pahor A, Sugg J, Rohwedder K, et al. Long-term efficacy of dapagliflozin in patients with type 2 diabetes mellitus receiving high doses of insulin: a randomized trial. Ann Intern Med. 2012;156(6):405-15.

15. Sonesson C, Johansson PA, Johnsson E, Gause-Nilsson I. Cardiovascular effects of dapagliflozin in patients with type 2 diabetes and different risk categories: a meta-analysis. Cardiovasc Diabetol. 2016;15(1):37.

16. Ptaszynska A, Johnsson KM, Parikh SJ, de Bruin TW, Apanovitch AM, List JF. Safety profile of dapagliflozin for type 2 diabetes: pooled analysis of clinical studies for overall safety and rare events. Drug Saf. 2014;37:815-29.

17. EMA. Forxiga (dapagliflozin): summary of product characteristics. 2014. Available from: http://www.ema.europa.eu/docs/ en_GB/document_library/EPAR_-_Product_Information/human/ 002322/WC500136026.pdf. Accessed 24 Feb 2016.

18. Bailey CJ, Gross JL, Pieters A, Bastien A, List JF. Effect of dapagliflozin in patients with type 2 diabetes who have inadequate glycaemic control with metformin: a randomised, doubleblind, placebo-controlled trial. Lancet. 2010;375(9733):2223-33.

19. Bailey CJ, Iqbal N, T'Joen C, List JF. Dapagliflozin monotherapy in drug-naive patients with diabetes: a randomized-controlled trial of low-dose range. Diabetes Obes Metab. 2012;14(10):951-9.

20. Bolinder J, Ljunggren $\mathrm{O}$, Kullberg J, Johansson L, Wilding J, Langkilde AM, et al. Effects of dapagliflozin on body weight, total fat mass, and regional adipose tissue distribution in patients with type 2 diabetes mellitus with inadequate glycemic control on metformin. J Clin Endocrinol Metab. 2012;97(3):1020-31.

21. Cefalu WT, Leiter LA, de Bruin TW, Gause-Nilsson I, Sugg J, Parikh SJ. Dapagliflozin's effects on glycemia and cardiovascular risk factors in high-risk patients with type 2 diabetes: a 24-week, multicenter, randomized, double-blind, placebo-controlled study with a 28-week extension. Diabetes Care. 2015;38(7):1218-27.

22. Ferrannini E, Ramos SJ, Salsali A, Tang W, List JF. Dapagliflozin monotherapy in type 2 diabetic patients with inadequate glycemic control by diet and exercise: a randomized, doubleblind, placebo-controlled, phase 3 trial. Diabetes Care. 2010;33(10):2217-24.

23. Jabbour SA, Hardy E, Sugg J, Parikh S. Dapagliflozin is effective as add-on therapy to sitagliptin with or without metformin: a 24-week, multicenter, randomized, double-blind, placebo-controlled study. Diabetes Care. 2014;37(3):740-50.

24. Kaku K, Inoue S, Matsuoka O, Kiyosue A, Azuma H, Hayashi N, et al. Efficacy and safety of dapagliflozin as a monotherapy for type 2 diabetes mellitus in Japanese patients with inadequate glycaemic control: a phase II multicentre, randomized, doubleblind, placebo-controlled trial. Diabetes Obes Metab. 2013;15(5): 432-40.

25. Kohan DE, Fioretto P, Tang W, List JF. Long-term study of patients with type 2 diabetes and moderate renal impairment shows that dapagliflozin reduces weight and blood pressure but does not improve glycemic control. Kidney Int. 2014;85(4):962-71.

26. Lambers Heerspink HJ, de Zeeuw D, Wie L, Leslie B, List J. Dapagliflozin a glucose-regulating drug with diuretic properties in subjects with type 2 diabetes. Diabetes Obes Metab. 2013;15(9):853-62.

27. Leiter LA, Cefalu WT, de Bruin TW, Gause-Nilsson I, Sugg J, Parikh SJ. Dapagliflozin added to usual care in individuals with type 2 diabetes mellitus with preexisting cardiovascular disease: a 24-week, multicenter, randomized, double-blind, placebo-controlled study with a 28-week extension. J Am Geriatr Soc. 2014;62(7):1252-62.

28. List JF, Woo V, Morales E, Tang W, Fiedorek FT. Sodiumglucose cotransport inhibition with dapagliflozin in type 2 diabetes. Diabetes Care. 2009;32(4):650-7.
29. Weber MA, Mansfield TA, Alessi F, Iqbal N, Parikh S, Ptaszynska A. Dapagliflozin effects on blood pressure in hypertensive diabetic patients on renin-angiotensin system blockade. Blood Press. 2016;25(2):93-103.

30. Mudaliar S, Henry RR, Boden G, Smith S, Chalamandaris AG, Duchesne D, et al. Changes in insulin sensitivity and insulin secretion with the sodium glucose cotransporter 2 inhibitor dapagliflozin. Diabetes Technol Ther. 2014;16(3):137-44.

31. Wilding JP, Norwood P, T'Joen C, Bastien A, List JF, Fiedorek FT. A study of dapagliflozin in patients with type 2 diabetes receiving high doses of insulin plus insulin sensitizers: applicability of a novel insulin-independent treatment. Diabetes Care. 2009;32(9):1656-62.

32. Henry RR, Murray AV, Marmolejo MH, Hennicken D, Ptaszynska A, List JF. Dapagliflozin, metformin XR, or both: initial pharmacotherapy for type 2 diabetes, a randomised controlled trial. Int J Clin Pract. 2012;66(5):446-56.

33. Levey AS, Bosch JP, Lewis JB, Greene T, Rogers N, Roth D. A more accurate method to estimate glomerular filtration rate from serum creatinine: a new prediction equation. Modification of Diet in Renal Disease Study Group. Ann Intern Med. 1999;130(6): 461-70.

34. Komoroski B, Vachharajani N, Feng Y, Li L, Kornhauser D, Pfister M. Dapagliflozin, a novel, selective SGLT2 inhibitor, improved glycemic control over 2 weeks in patients with type 2 diabetes mellitus. Clin Pharmacol Ther. 2009;85(5):513-9.

35. Nauck MA. Update on developments with SGLT2 inhibitors in the management of type 2 diabetes. Drug Des Devel Ther. 2014;8:1335-80.

36. Geerlings S, Fonseca V, Castro-Diaz D, List J, Parikh S. Genital and urinary tract infections in diabetes: impact of pharmacologically-induced glucosuria. Diabetes Res Clin Pract. 2014;103(3): 373-81.

37. Hirji I, Guo Z, Andersson SW, Hammar N, Gomez-Caminero A. Incidence of urinary tract infection among patients with type 2 diabetes in the UK General Practice Research Database (GPRD). J Diabetes Complications. 2012;26(6):513-6.

38. Abdel-Kader K, Palevsky PM. Acute kidney injury in the elderly. Clin Geriatr Med. 2009;25(3):331-58.

39. Sharabi Y, Illan R, Kamari Y, Cohen H, Nadler M, Messerli FH, et al. Diuretic induced hyponatraemia in elderly hypertensive women. J Hum Hypertens. 2002;16(9):631-5.

40. Thomas MC. Renal effects of dapagliflozin in patients with type 2 diabetes. Ther Adv Endocrinol Metab. 2014;5(3):53-61.

41. Schmitt R, Coca S, Kanbay M, Tinetti ME, Cantley LG, Parikh CR. Recovery of kidney function after acute kidney injury in the elderly: a systematic review and meta-analysis. Am J Kidney Dis. 2008;52(2):262-71.

42. Abuelo JG. Normotensive ischemic acute renal failure. N Engl J Med. 2007;357(8):797-805.

43. Arampatzis S, Funk GC, Leichtle AB, Fiedler GM, Schwarz $\mathrm{C}$, Zimmermann $\mathrm{H}$, et al. Impact of diuretic therapy-associated electrolyte disorders present on admission to the emergency department: a cross-sectional analysis. BMC Med. 2013;11:83.

44. Ljunggren O, Bolinder J, Johansson L, Wilding J, Langkilde AM, Sjostrom CD, et al. Dapagliflozin has no effect on markers of bone formation and resorption or bone mineral density in patients with inadequately controlled type 2 diabetes mellitus on metformin. Diabetes Obes Metab. 2012;14(11):990-9.

45. Perazella MA, Mahnensmith RL. Hyperkalemia in the elderly: drugs exacerbate impaired potassium homeostasis. J Gen Intern Med. 1997;12(10):646-56.

46. Zinman B, Wanner C, Lachin JM, Fitchett D, Bluhmki E, Hantel $\mathrm{S}$, et al. Empagliflozin, cardiovascular outcomes, and mortality in type 2 Diabetes. N Engl J Med. 2015;373(22):2117-28. 
47. Weber MA, Mansfield TA, Cain VA, Iqbal N, Parikh SJ, Ptaszynska A. Blood pressure and glycaemic effects of dapagliflozin in patients with type 2 diabetes on combination antihypertensive therapy: a randomised, placebo-controlled study. Lancet Diabetes Endocrinol. 2016;4(3):211-20.

48. Dziuba J, Alperin P, Racketa J, Iloeje U, Goswami D, Hardy E, et al. Modeling effects of SGLT-2 inhibitor dapagliflozin treatment versus standard diabetes therapy on cardiovascular and microvascular outcomes. Diabetes Obes Metab. 2014;16(7): 628-35.

49. Ptaszynska A, Hardy E, Johnsson E, Parikh S, List J. Effects of dapagliflozin on cardiovascular risk factors. Postgrad Med. 2013;125(3):181-9. 